\title{
Editorial
}

\section{Duration of chemotherapy in small cell lung cancer}

Chemotherapy for small cell lung cancer was associated with considerable optimism in the mid 1970s, when an increasing duration of median survival was achieved with combination chemotherapy. Radiation therapy of the primary tumour site and mediastinum was commonly added before or between chemotherapy cycles in an attempt to improve tumour control at the primary site. A decade later it is proving difficult to improve the duration of survival with currently available medication and further advances will depend on the emergence of new agents and approaches as a result of the great increase in our understanding of the biology of the tumour.

In studies of unselected patients those presenting with limited disease (that is, confined to one hemithorax and the ipsilateral supraclavicular fossa) have a $30-50 \%$ rate of complete response to chemotherapy, with a median survival of 10-16 months. Among patients presenting with extensive disease (including at least one extrathoracic site) the complete response rate is $15-20^{\circ}{ }_{0}$ and the median survival 6-12 months. ${ }^{12}$ Early studies on selected patients suggested that an appreciable proportion might be cured, ${ }^{3}$ but analysis of patients alive two years from diagnosis and treatment gives less hopeful results. Davis et $a l^{4}$ reported on 1580 cases of small cell lung cancer in general hospital practice in Seattle and found only $2 \cdot 4^{\circ}{ }_{0}$ alive at five years. Of 874 patients treated at the Finsen Institute in Copenhagen, only $7 \cdot 6^{\circ}$ o were alive at 18 months. ${ }^{5}$ Five years after the end of treatment the risk of relapse from small cell lung cancer had reached $46^{\circ}{ }_{0}$ in this group of survivors, and the risk of dying from a second cancer (usually a non-small cell lung cancer) had risen to $32^{\circ}{ }_{0}$. Thirty eight of 54 disease free survivors, however, were able to resume their prediagnosis life style and occupation. In Britain an analysis of 3681 patients entered into clinical trials showed a two year survival of $8.5^{\circ}$, for those presenting with limited disease and $2 \cdot 2^{\circ}{ }_{0}$, for those with extensive disease. ${ }^{6}$

Attempts to improve both the median and the long term survival for patients with small cell lung cancer have included various strategies. "Maintenance" chemotherapy has been given after the initial induction period with cyclical chemotherapy, the same or different drugs being given either at lower dose or at longer intervals in an attempt to prolong the duration of remission. Doses have been increased beyond conventional levels, autologous bone marrow transplantation sometimes being used to shorten the period of aplasia. This has been done either as initial induction treatment or as "late intensification" to consolidate or prolong remission. The use of cyclical alternating combinations of drugs considered to be noncross resistant has been assessed and new drugs and new drug combinations in intensive regimens have continued to be explored.

None of these approaches has yet made a major impact. Newer agents such as etoposide, teniposide, ifosfamide, cisplatin, and carboplatin have been shown to be highly active as single agents or in combination (for example, etoposide and cisplatin). These drugs are used in many chemotherapy regimens, but no consistent improvement in median or long term survival has yet been found in unselected cases.

There has been a disappointing lack of impact from the addition of radiotherapy to chemotherapy. Although radiotherapy reduces the likelihood of recurrence at the primary site, it has had little effect on survival because chemotherapy has not controlled disseminated disease adequately. Despite different dose schedules for radiotherapy and attempts to administer the treatment at different stages during the course of chemotherapy, the effect on survival in large studies is small. ${ }^{7}$

In view of this lack of progress, some recent studies have assessed the optimal duration of administration of chemotherapy. Undoubtedly most patients respond to chemotherapy with considerable improvement in terms of presenting symptoms and of measures that attempt to assess the quality of life. Most patients, however, especially the majority who have extensive disease, obtain only short remissions before disease progression and death. The balance between effective treatment and minimal toxicity is therefore crucial.

Several studies have addressed the question of the value of maintenance chemotherapy after an initial response, and also the length of initial chemotherapy with or without further chemotherapy when relapse occurs. In 1984 Feld $e t$ $a l^{8}$ published two sequential non-randomised studies. The first was concerned with 147 patients treated by three courses of cyclophosphamide, Adriamycin (doxorubicin), and vincristine (CAV); thoracic irradiation; and then a year of maintenance chemotherapy with lomustine, procarbazine, and methotrexate. Their second study was of 320 patients (153 limited disease and 167 extensive disease) treated by six courses of CAV and thoracic irradiation but no maintenance chemotherapy. In the latter study the median survival time was 49 weeks for limited disease and 34 weeks for extensive disease, similar to survival in the initial study, which included one year of maintenance chemotherapy.

Two large randomised studies on the duration of chemotherapy have recently been published. Our group ${ }^{9}$ randomised 616 patients to receive either four or eight courses of chemotherapy and at relapse to receive either symptomatic treatment or further chemotherapy using agents other than those used for initial chemotherapy. The study showed that patients allocated to receive four courses of initial chemotherapy only (with no further chemotherapy at relapse) had a median survival of 30 weeks. This was significantly inferior to the other three treatment arms, which had median survivals of 39-42 weeks. The difference was greater for the responding populations. We concluded that if only four courses of chemotherapy were given there was a survival disadvantage unless patients received chemotherapy at relapse, but if eight courses were given initially then there was no advantage in further chemotherapy at relapse. The policy of chemotherapy on relapse was, however, difficult to achieve because patients and physicians were reluctant to start chemotherapy again. 
In the Medical Research Council (MRC) study ${ }^{10} 497$ patients were given six cycles of a four drug regimen with additional radiotherapy for responding patients with limited disease between courses 2 and 3. At the completion of the initial six cycles patients whose disease remained controlled were randomly allocated to two groups, receiving either no further chemotherapy or six further courses of the same drugs but at slightly longer intervals (four weeks instead of three weeks). The overall response rate was $66 \%$, similar to that of our study $(61 \%)$. The median survival of all patients entered was also 39 weeks. There was no overall survival advantage for patients who received the maintenance treatment, though for the 97 patients who were complete responders after initial chemotherapy there was a suggestion of longer survival with maintenance treatment -42 weeks from entry to the study against 30 weeks for those not given maintenance chemotherapy. An attempt was made in a small proportion of the patients to assess the quality of their lives during treatment. This suggested that maintenance treatment was associated with toxicity and side effects, as assessed by physician and patient, and with a poorer quality of life.

Few studies have addressed the question of quality of life during chemotherapy and afterwards. A further study by our group evaluated eight measures designed to assess the quality of life and compared them in patients receiving four and eight cycles during the second part of the treatment, when they were receiving courses $5-8$ or no further treatment. All the quality of life measures showed deterioration during courses $5-8$, increasingly so as treatment progressed. This adverse consequence of the second four courses of chemotherapy, however, has to be set against the longer median survival seen with eight courses of chemotherapy than with four courses. In a separate study chemotherapy also showed a considerable initial benefit in terms of quality of life during treatment, about $65 \%$ of those with symptoms obtaining relief from pain, cough, shortness of breath, superior vena caval obstruction, or dysphagia. ${ }^{11}$

Two other large studies were presented at the 5 th World Lung Cancer Conference in August 1988. Three hundred and twenty patients received six cycles of lomustine, cyclophosphamide, doxorubicin, and etoposide. The 79 complete responders were randomly allocated to groups receiving either no further treatment or another six cycles of chemotherapy. ${ }^{12}$ No difference in median survival was found in these patients. The median survival was 65 weeks for those having further chemotherapy with a two year survival rate of $24 \%$, and 60 weeks for those having no further chemotherapy with a two year survival of $27 \%$.

A large European Organisation for Research and Treatment of Cancer (EORTC) study ${ }^{13}$ included 426 patients who received five courses of cyclophosphamide, doxorubicin, and etoposide. Responders were then randomly allocated to conservative treatment only or a further seven courses of the same chemotherapy. Again there was no survival advantage for either group. The study did, however, show an increased time to relapse with maintenance chemotherapy, but this did not affect median survival.
What conclusions can be gleaned from these studies? It would seem reasonable to conclude that around six courses of chemotherapy is a reasonable optimum duration for initial chemotherapy and that maintenance chemotherapy, or further treatment at relapse, is not useful. The question still remains of whether all patients with small cell lung cancer should be treated for this period. For patients presenting with extensive disease this would appear to be a reasonable decision. The median survival would be acceptable by current standards, as would toxicity and the effects on quality of life. The MRC Lung Cancer Working Party is currently evaluating three versus six courses of chemotherapy, though to judge from our study, where four courses appeared less than optimal in terms of median survival, three courses are unlikely to be as effective as six.

Should all patients with limited disease now be offered short duration chemotherapy? More aggressive treatment of patients with limited disease and smaller tumour volume has not yet been shown to prolong survival. New drugs and new methods of intensification of treatment may prolong survival in patients with a good prognosis and these are being explored by several groups; but until these treatments have been assessed in controlled trials there appears to be no benefit in prolonging chemotherapy in small cell lung cancer, even in those with limited disease.

Consultant Physician and Honorary Senior Lecturer, Brompton Hospital, University College Hospital, and Middlesex Hospital, London

R L SOUHAMI

Kathleen Ferrier Professor of Oncology, University College and Middlesex School of Medicine, London

Address for reprint requests: Dr S G Spiro, Brompton Hospital, London SW3 6HP.

1 Spiro SG. Chemotherapy of small cell lung cancer. In: Spiro SG, ed. Clinics in oncology. Vol 4. London: Saunders, 1985:105-20.

2 Bunn PA, Ihde DC. Small cell bronchogenic carcinoma: a review of therapeutic results. In: Livingstone RB, ed. Lung cancer. Vol 1. The Hague: Martinus Nijhoff, 1981:169-208.

3 Greco FA, Richardson FL, Oldham RK. Small cell lung cancer. Complete remission and improved survival. Am J Med 1979;66:625-30.

4 Davis S, Wright PW, Schulman SF, Scholes D, Thorning D, Hammar S. Long-term survival in small cell carcinoma of the lung. A population experience. J Clin Oncol 1985;3:80-91.

5 Osterlind K, Hansen HH, Dombernowsky P. Mortality and morbidity in long-term surviving patients treated with chemotherapy with or without irradiation for small cell lung cancer. J Clin Oncol 1986;4:1044-52.

6 Souhami RL, Law K. Longevity in small cell lung cancer. Br J Cancer (in press).

7 Perry MG, Eaton WL, Propert KJ, et al. Chemotherapy with or without radiation therapy in limited small cell carcinoma of the lung. $N$ Engl J Med radiation therapy in

8 Feld R, Evans WK, DeBoer G, et al. Combined modality induction therapy without maintenance chemotherapy for small cell carcinoma of the lung. $J$ Clin Oncol 1984;2:294-304.

9 Spiro SG, Souhami RL, Geddes DM, et al. Duration of chemotherapy in small cell lung cancer: a Cancer Research Campaign trial. Br J Cancer 1989;59:578-83.

10 Medical Research Council Lung Cancer Working Party. Controlled trial of twelve versus six courses of chemotherapy in the treatment of small cell lung cancer. Br J Cancer 1989;59:584-90.

11 Geddes DM. Quality of life. In: Spiro SG, ed. Clinics in oncology. Vol 4. London: Saunders, 1985:161-76.

12 Lebeau B, Chastang C, Capron F, and the "Petites Cellules" group. Small cell lung cancer (SCLC): first analysis of a randomised clinical trial of $6 \mathrm{vs}$ 12 chemotherapy cycles for complete responders [abstract]. Lung Cancer 12 chemotherapy

13 Splinter TAW. EORTC 08825: induction vs induction plus maintenance chemotherapy (CT) in small cell lung cancer (SCLC). Definitive evaluation [abstract]. Lung Cancer 1988;4:A100. 